\title{
Oppositionality and Socioemotional Competence: Interacting Risk Factors in the Development of Childhood Conduct Disorder Symptoms
}

William Mandy, D.Clin.Psy., David Skuse, M.D., Colin Steer, M.Sc., Beate St Pourcain, Ph.D., Bonamy R. Oliver, Ph.D.

\begin{abstract}
Objectives: Oppositional behavior in childhood is a probabilistic risk factor for the subsequent development of more serious conduct problems characteristic of conduct disorder (CD). The capacity to understand the subjective states of others (socioemotional competence) helps regulate antisocial behavior in typical development. We hypothesized that socioemotional competence moderates the developmental relationship between oppositionality and CD symptoms, such that oppositional defiant disorder (ODD) symptoms pose the greatest risk for subsequent $C D$ symptoms in children with poor socioemotional competence. Method: Parent-report data were collected for 6,218 children at 7 and 10 years of age. Bootstrap multiple regression predicting CD symptoms at age 10 was used to test for an interaction between socioemotional competence and ODD symptoms, while also accounting for direct effects and controlling for sex, maternal education, attention-deficit/hyperactivity disorder symptoms, and $\mathrm{CD}$ symptoms at 7 years. We further tested whether the interaction applied to both males and females, and to both aggressive and rule-breaking CD symptoms. Results: A significant interaction was found between ODD and socioemotional competence: the association between oppositionality at 7 years and CD traits at 10 years was strongest for children with poor socioemotional capacities. As predicted, this moderation effect was significant in a model predicting aggression, but it was not significant for rulebreaking CD symptoms. Conclusion: Socioemotional competence moderates the developmental relationship between mid-childhood oppositionality and more serious conduct problems in later childhood. A capacity to understand the subjective states of others may buffer the risk posed by oppositionality for later CD symptoms, including aggression. J. Am. Acad. Child Adolesc. Psychiatry, 2013;52(7):718-727. Key Words: Avon Longitudinal Study of Parents and Children (ALSPAC), conduct disorder (CD), developmental psychopathology, oppositional defiant disorder (ODD), socioemotional competence
\end{abstract}

O ppositional defiant disorder (ODD) is characterized by "a pattern of negativistic, defiant, disobedient, or hostile behavior toward authority figures" (p. 100), ${ }^{1}$ whereas the cardinal feature of conduct disorder (CD) is a "repetitive and persistent pattern of behavior in which the basic rights of others or major age appropriate societal norms are violated" (p. 93). ${ }^{1}$ Both of these childhood disruptive behavior disorders are dimensional, ${ }^{2}$ each representing the extreme of distinct trait distributions that extend throughout the general population. ${ }^{3}$

Elevated ODD symptoms are a risk factor for the subsequent development of $\mathrm{CD}$ and $\mathrm{CD}$ symptoms, ${ }^{4,5}$ yet this relationship is probabilistic and complex, as illustrated by the fact that most children with ODD do not go on to develop CD. ${ }^{6}$ One approach to understanding the variability of outcomes in development draws upon a core principle of developmental psychopathology: that individual differences in adaption and maladaption arise from the interaction of multiple risk and protective factors. ${ }^{7}$ On this basis, the investigation of variables that moderate the influence of ODD traits may help to explain continuities and discontinuities with later CD symptoms.

Conduct problems, especially those with childhood onset, are frequently associated with 
abnormalities in social cognition. ${ }^{8}$ In particular, children with a callous and unemotional interpersonal style are at risk for developing the severe and persistent externalizing problems characteristic of childhood-onset CD. ${ }^{9}$ Other forms of atypical social cognition, such as impaired emotion recognition ${ }^{10}$ and poor theory of mind. ${ }^{11}$ have also been implicated in the development of childhood-onset CD. Some evidence exists that elements of social cognition moderate the development of childhood conduct problems, by amplifying or attenuating the effects of other risk factors. For example, in early childhood, low maternal emotional support is only predictive of increases in aggression in children who have delayed theory of mind development. ${ }^{12}$ Moreover, callous-unemotional traits weaken the relationship between poor parenting and childhood disruptive behavior problems, ${ }^{13}$ and strengthen the association between initial and subsequent CD symptoms. ${ }^{6}$

In the current study, we tested a novel social moderation hypothesis by considering whether the capacity to accurately perceive the perspectives and emotions of others (termed socioemotional competence) influences the developmental relationship between ODD and CD traits in childhood. This was based on clinical experience and the 3 following related arguments. First, ODD is characterized by emotional and behavioral dysregulation that places children at risk for committing the transgressive acts symptomatic of $\mathrm{CD} .^{14}$ Second, these acts, particularly those involving direct aggression (e.g., fighting, bullying, threatening), engender negative affect in others. Third, a capacity for perceiving this affect offers an opportunity for "top-down" regulation of behavior by making an aversive experience available to a child who has caused distress or anger. ${ }^{15,16}$ Conversely, those with limited socioemotional competence are, by definition, less likely to notice the negative impact of their behavior on others, and thus miss out on this form of interpersonal behavioral regulation.

Accordingly, we predicted that children with ODD symptoms and poor socioemotional competence in middle childhood would be at elevated risk for developing CD symptoms, and, conversely, that children who are oppositional, but who show intact socioemotional competence, would be at lesser risk for developing CD symptoms.

Beyond this overarching hypothesis, we were interested in 2 additional issues. The first was based on factor analytic, phenotypic, and behavior genetic findings suggesting that symptoms of $C D$ can be divided into 2 related but meaningfully distinct "aggressive" and "rule-breaking" clusters. ${ }^{17}$ We predicted that the moderating effect of socioemotional competence would be most pertinent to overt, aggressive CD symptoms (such as fighting, bullying and threatening), as these are more likely to elicit immediate, observable emotional distress in others compared to more covert, rule-breaking $\mathrm{CD}$ behaviors (lying, stealing, staying out late, playing truant). Second, because boys are more likely to progress from ODD to CD than are girls, ${ }^{18}$ it is possible that sex influences developmental processes that link oppositionality and later $C D$ behaviors. For this reason, we tested whether the moderation effect that we predicted applied to both boys and girls.

Because attention-deficit/hyperactivity disorder (ADHD) symptoms are a risk factor for a range of conduct problems ${ }^{4}$ and, as such, may carry some of the cross-sectional and developmental association between ODD and CD, we also included measures of inattention and hyperactivityimpulsivity in our analyses to control for these potential confounds. We tested our hypotheses using parent-report measures in a large, UK community sample of children assessed at 7 and 10 years of age.

\section{METHOD}

\section{Sample}

Participants were members of the Avon Longitudinal Study of Parents and Children (ALSPAC), a populationbased, longitudinal cohort in southwestern England, comprising individuals with a predicted birth date between April 1, 1991, and December 31, 1992. The initial cohort included 14,541 pregnancies, with 13,971 children alive at 12 months. Ethical approval was obtained from the ALSPAC Law and Ethics Committee and Local Research Ethics Committees. Cohort members were included in the current study if they had complete behavioral data required to test the study hypotheses (see Data Analysis). Demographic characteristics for the 6,218 children who were eligible are presented in Table 1. In comparison to participants in the current study, those in the original ALSPAC cohort excluded from our analyses were more likely to have a mother who was adolescent (odds ratio [OR] $=5.15,95 \%$ confidence interval [CI] 4.13-6.43), had no higher or further education $(\mathrm{OR}=1.81,95 \% \mathrm{CI}=1.70-1.95)$, and was not a home owner $(\mathrm{OR}=3.15,95 \% \mathrm{CI}=2.90-3.43)$.

\section{Measures}

Development and Well-Being Assessment (DAWBA). ODD, $\mathrm{CD}$, and $\mathrm{ADHD}$ symptoms were measured using the DAWBA, administered as a parent-report questionnaire 
TABLE 1 Characteristics of the Sample $(N=6,128)$

\begin{tabular}{lc}
\hline Characteristics \\
Age in months at time 1, mean (SD) & $91.7(1.4)$ \\
Age in months at time 2, mean (SD) & $128.6(1.6)$ \\
Proportion female, \% & 49.0 \\
Proportion with white ethnicity, \% & 95.1 \\
Proportion born to adolescent mother, \% & 1.5 \\
Proportion with mother with a university & 17.5 \\
$\quad$ degree, \% & \\
Proportion with parent(s) who own their & 84.6 \\
home, \%
\end{tabular}

when participating children were 7 and 10 years old. The DAWBA contains symptom checklists in which each item corresponds to a DSM-IV and ICD-10 diagnostic criterion, and has been extensively validated in community samples. ${ }^{19}$ For each ODD and ADHD item, parents were asked to rate whether, "in the last 6 months and compared to other children of the same age," their child had had the symptom "no more than others," "a little more than others," or "a lot more than others." For CD, parents were asked whether their child had shown each symptom within the last 12 months, with the answer codes "no," "perhaps," and "definitely." ODD, ADHD, and CD symptom scores were calculated by summing DAWBA items. The ODD scale comprised 9 items, which were summed to give an overall oppositionality score. The overall $\mathrm{CD}$ symptoms scale was the sum of 7 DAWBA items, comprising the most commonly reported symptoms of $\mathrm{CD}$. More extreme manifestations of $\mathrm{CD}$, including sexual assault and torturing animals, were not included in ALSPAC questionnaires due to their rarity in the general population of children and to minimize the risk of increasing sample attrition. We summed clusters of DAWBA CD items to create aggressive ("has often bullied or threatened," "often started fights") and rulebreaking ("often told lies," "often stayed out after dark, much later than he was supposed to," "stolen from the house, or from other people's houses, or from shops or school," "run away from home more than once," "often played truant") CD symptom scales. DAWBA ADHD items were used to calculate separate scales for inattention and hyperactivity-impulsivity, each comprising 9 DAWBA items.

Socioemotional Competence. Socioemotional competence was measured at age 7 years using a subset of questions from the Social Communication Disorders Checklist. ${ }^{20}$ This 4 -item subscale, derived on the basis of face validity and factor analysis, has been used previously for examining the relationship between socioemotional competence and conduct problems. ${ }^{21}$ It is summed from parental responses to the following items: "Not aware of other people's feelings," "Does not realize when others are upset or angry," "Does not notice the effect of his/her behavior on other members of the family," and "Does not pick up on body language." Response codes are "not true," "quite or sometimes true," and "very or often true." This socioemotional competence scale excludes those items of the Social Communication Disorders Checklist that concern behavioral (e.g., "Child is very demanding of other people's time") or emotional (e.g., "Child is difficult to reason with when upset") aspects of social communication difficulties. This is for the purpose of achieving a focused measure of socioemotional competence less likely than the full Social Communication Disorders Checklist to yield scores influenced by externalizing and internalizing problems.

There is evidence for the construct validity of this socioemotional competence scale, as its pattern of associations with measures of other constructs is in line with theoretical expectations. Specifically, in terms of convergent validity, the scale is sensitive to diagnosed cases of autism spectrum disorder in the ALSPAC cohort and correlates moderately with scores on the Child Communication Checklist, a measure of pragmatic language. ${ }^{21,22}$ Also, as would be predicted for a scale of socioemotional competence, ${ }^{23}$ males score worse than females on the scale. Its discriminant validity is suggested by findings of weak correlations with distinct constructs, such as IQ, low socioeconomic status, and teenage pregnancy. ${ }^{21}$

Maternal Education. Maternal education was used as an indicator of socioeconomic variability, as this has been shown to be a valid index of socioeconomic status in the ALSPAC cohort. ${ }^{24}$ At 32 weeks gestation, mothers reported their highest education attainment as: "none"; "CSE" (basic level educational attainment in the UK); "vocational qualifications;" "O-level" (at the time of data collection, the minimum requirement for progression to further education); "A-levels" (highest school-based attainment typically awarded, aged 18 years); and "degree or above." For this variable, higher scores indicate higher maternal education.

\section{Data Analyses}

To test our moderation hypothesis, we used linear multiple regression to assess the significance of the interaction between ODD and socioemotional competence. In our main model, we used overall $C D$ symptom score at 10 years of age as the outcome variable, and included the following as predictors: maternal education; sex; CD symptoms at 7 years; ODD symptoms at 7 years; inattention at 7 years; hyperactivityimpulsivity at 7 years; socioemotional competence at 7 years; and the interaction term for socioemotional competence and ODD. This allowed us to test for an interaction between socioemotional competence and ODD, while accounting for main effects of ODD traits and socioemotional competence and controlling for maternal education, sex, ADHD traits, and CD symptomsat age 7 years. To test the expectation that the interaction between socioemotional competence and oppositionality would be most relevant to the development of aggressive rather than rule-breaking conduct 
symptoms, we ran 2 further regression models with identical predictors to those described above, but with aggressive and rule-breaking CD symptoms as outcome variables. To investigate differences in interaction effects between these 2 models, multivariate linear regression was used.

To test for sex differences in moderation effects, we ran 3 additional regression models with overall $C D$, aggressive $C D$, and rule-breaking $C D$ as their respective outcome variables. For each of these models, in addition to the predictor variables described above, we entered a 3-way interaction term for socioemotional competence $\times$ ODD $\times$ sex. To avoid making any assumptions concerning the other potential 2-way interactions (socioemotional competence $\times$ sex, ODD $\times$ sex), these terms were also fitted.

To ensure robustness of our parameter estimates, given the deviations from normality for our outcomes, we used bootstrap regression based on 10,000 random samples (with replacement) of size $n$, where $n$ reflects the number of children with complete data for each outcome separately. This allowed empirically based $95 \%$ confidence intervals and $p$ values to be derived, and avoided any normality-based assumption associated with the t-distribution used in standard linear regression. To illustrate the interaction effects (Figure 1), we divided the sample into 3 groups based on degree of socioemotional competence: no socioemotional competence difficulties $(\mathrm{n}=3,832) ; 1$ difficulty $(\mathrm{n}=1,140)$; and 2 or more difficulties $(n=1,246)$. A difficulty was considered present if a parent answered "quite or sometimes true" or "very or often true" to an item on the socioemotional competence scale. For each group, we used regression to examine the relationship between ODD traits at 7 years of age and CD symptoms at 10 years, controlling for sex, maternal education, and CD and ADHD symptoms at 7.

\section{RESULTS}

Bivariate correlations with bootstrap 95\% confidence intervals for continuous variables used in our regression analyses are shown in Table 2 . Bootstrap $t$ tests were used to test for sex differences on key variables. Boys (mean $=3.29$, $\mathrm{SD}=$ $1.19)$ and girls (mean $=3.31, \mathrm{SD}=1.21)$ did not differ on maternal education $(p=.539)$. Boys (mean $=1.55, \mathrm{SD}=3.04)$ scored higher than girls (mean $=1.13, \mathrm{SD}=2.42$ ) for ODD traits at age 7 years $(p<.001)$. At both time points in the study, boys (age 7 years, mean $=0.59, \mathrm{SD}=1.07$; age 10 years, mean $=0.54, \mathrm{SD}=1.12)$ had more $(p<$ .001) CD symptoms than girls (age 7 years, mean $=0.49$, $\mathrm{SD}=0.92$; age 10 years, mean $=$ $0.39, \mathrm{SD}=0.87$ ). On average, girls (mean $=0.71$, $\mathrm{SD}=1.24)$ had superior $(p<.001)$ socioemotional competence to that of boys (mean $=0.97$, $\mathrm{SD}=1.52)$.

Moderation by Socioemotional Competence of the Relationship Between Oppositionality and Subsequent CD Symptoms

Table 3 shows standardized beta coefficents $(\beta)$ for the bootstrap regression models designed to

FIGURE 1 Association between oppositionality at age 7 years and conduct disorder (CD) symptoms at 10 years for groups defined by degree of socioemotional competence. Note: All analyses control for sex and maternal education, as well as attention-deficit/hyperactivity disorder and conduct disorder symptoms at 7 years.

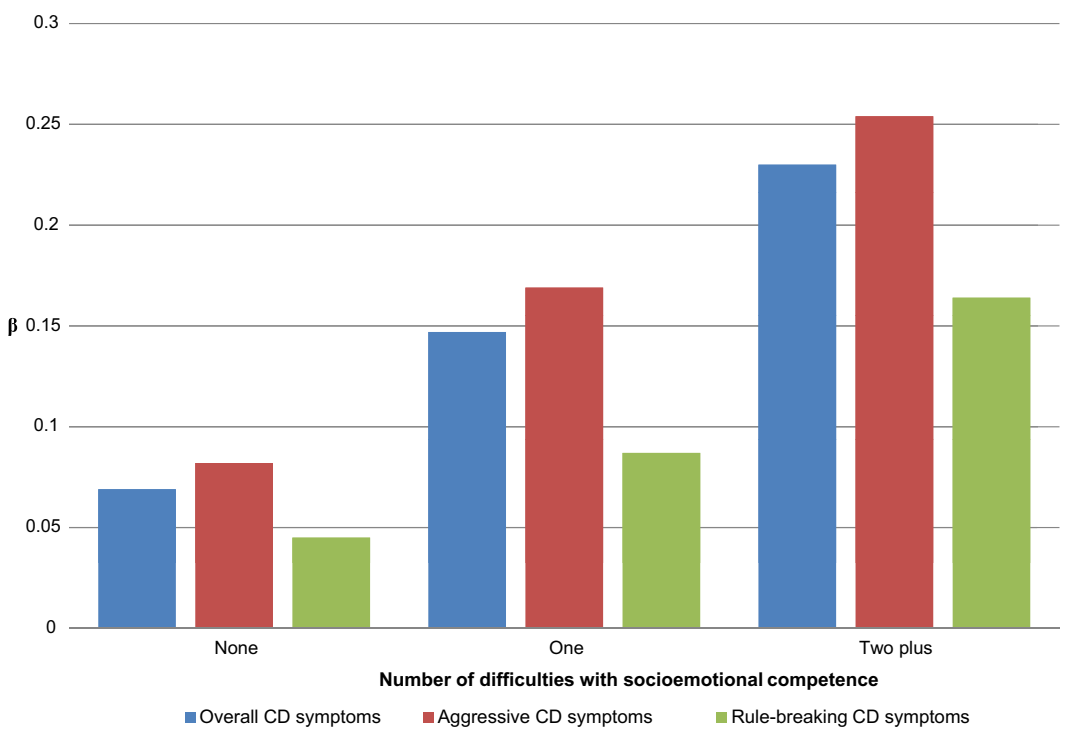


TABLE 2 Bootstrap Bivariate Correlations Between Study Variables

\begin{tabular}{|c|c|c|c|c|c|c|c|c|}
\hline \multirow[b]{2}{*}{ Characteristic } & \multicolumn{8}{|c|}{ R $(95 \% \mathrm{Cl})$} \\
\hline & 2 & 3 & 4 & 5 & 6 & 7 & 8 & 9 \\
\hline 1 Maternal & $-0.03 * * *$ & $-0.07^{* * *}$ & $-0.04^{* *}$ & $-0.03^{*}$ & $-0.07^{* * *}$ & $-0.08 * * *$ & $-0.06 * * *$ & $-0.08 * * *$ \\
\hline education & $(-0.06$ to -0.01$)$ & $(-0.09$ to -0.04$)$ & $(-0.06$ to -0.01$)$ & $(-0.06$ to -0.01$)$ & $(-0.10$ to -0.05$)$ & $(-0.11$ to -0.06$)$ & $(-0.09$ to -0.03$)$ & $(-0.10$ to -0.05$)$ \\
\hline 2 ODD symptoms & - & $0.45^{* * *}$ & $0.46^{* * *}$ & $0.49 * * *$ & $0.58^{* * *}$ & $0.36^{* * *}$ & $0.33^{* * *}$ & $0.29 * * *$ \\
\hline at age 7 years & & $(0.42$ to 0.49$)$ & (0.43 to 0.49 ) & (0.46 to 0.52$)$ & $(0.55$ to 0.60$)$ & $(0.32$ to 0.40$)$ & $(0.28$ to 0.37$)$ & (0.25 to 0.33 ) \\
\hline 3 CD symptoms & - & - & $0.32^{* * *}$ & $0.34^{\star * *}$ & $0.38^{* * *}$ & $0.45^{* * *}$ & $0.33^{* * *}$ & $0.40 * * *$ \\
\hline at age 7 years & & & (0.29 to 0.35$)$ & (0.31 to 0.37$)$ & (0.35 to 0.42$)$ & (0.41 to 0.48$)$ & $(0.29$ to 0.38$)$ & (0.37 to 0.44$)$ \\
\hline 4 SEC at age & - & - & - & $0.41^{* \star *}$ & $0.45^{\star \star *}$ & $0.26^{* * *}$ & $0.21^{* * *}$ & $0.22^{* * *}$ \\
\hline 7 years & & & & (0.38 to 0.44$)$ & (0.42 to 0.48$)$ & (0.22 to 0.29$)$ & $(0.17$ to 0.25$)$ & (0.19 to 0.26$)$ \\
\hline $\begin{array}{c}5 \text { ADHD-inattentive } \\
\text { symptoms at } \\
\text { age } 7 \text { years }\end{array}$ & - & - & - & - & $\begin{array}{c}0.72^{* * *} \\
(0.70 \text { to } 0.74)\end{array}$ & $\begin{array}{c}0.29^{* * *} \\
(0.26 \text { to } 0.33)\end{array}$ & $\begin{array}{c}0.22^{* * *} \\
(0.18 \text { to } 0.26)\end{array}$ & $\begin{array}{c}0.26^{* * *} \\
(0.23 \text { to } 0.30)\end{array}$ \\
\hline $\begin{array}{l}6 \text { ADHD } \\
\text { hyperactive- } \\
\text { impulsive } \\
\text { symptoms at } \\
7 \text { years }\end{array}$ & - & - & - & - & - & $\begin{array}{c}0.33^{* * *} \\
(0.29 \text { to } 0.36)\end{array}$ & $\begin{array}{c}0.27^{* * *} \\
(0.23 \text { to } 0.30)\end{array}$ & $\begin{array}{c}0.28^{* * *} \\
(0.25 \text { to } 0.32)\end{array}$ \\
\hline $\begin{array}{c}7 \text { CD symptoms at } \\
\text { age } 10 \text { years }\end{array}$ & - & - & - & - & - & - & - & - \\
\hline $\begin{array}{c}8 \text { Aggressive CD } \\
\text { symptoms at } \\
10 \text { years }\end{array}$ & & & & & & & - & $\begin{array}{c}0.40 \\
(0.35 \text { to } 0.44)\end{array}$ \\
\hline $\begin{array}{l}9 \text { Rule-breaking CD } \\
\text { at } 10 \text { years }\end{array}$ & & & & & & & & - \\
\hline $\begin{array}{l}\text { Note: } A D H D=\text { attention }- \\
{ }^{p} p<.05, * * p<.0\end{array}$ & $\begin{array}{l}\text { yperactivity dis } \\
<.001 .\end{array}$ & $D=$ conduct dis & $D D D=o p$ & disor & $=$ socioemotional co & & & \\
\hline
\end{tabular}


test for an interaction between socioemotional competence and ODD in the prediction of CD symptoms at age 10 years. In the model predicting overall $C D$ symptoms, female sex and higher level of maternal education were associated with lower levels of CD symptoms at age 10 years. CD symptoms, inattention, and hyperactivity-impulsivity at age 7 years each independently predicted CD symptoms at age 10 , and there were no direct effects of ODD or socioemotional competence at age 7 in this model. There was a significant interaction between socioemotional competence and ODD $(p=.006)$. Figure 1 illustrates the nature of this interaction: the relationship between ODD at 7 years and CD at 10 years is stronger in the presence of socioemotional competence difficulties.

Our bootstrap regression model with aggressive $C D$ symptoms as an outcome variable shows that $C D$, inattention, hyperactivityimpulsivity, and ODD symptoms at age 7 , as well as male sex and lower maternal education, all predicted increased aggressive symptoms at age 10 . The interaction between socioemotional competence and ODD was significant $(p=.002)$. As shown in Figure 1, ODD had a stronger relationship with later aggressive $C D$ symptoms in children with socioemotional competence difficulties. In the rule-breaking CD model, overall CD symptoms, oppositionality, inattention, and hyperactivity-impulsivity at age 7 , and maternal education were all significant predictors. The interaction between socioemotional competence and ODD did not reach significance $(p=.079)$. There was no significant difference $(p=.092)$ in magnitude between the socioemotional $\times$ ODD interaction effects in our aggressive and rule-breaking models.

\section{Sex Differences}

To test whether our moderation hypothesis was equally applicable to boys and to girls, we ran further bootstrap regression models, adding to the models described in Table 3 a term for socioemotional competence $\times$ ODD $\times$ sex, along with terms for ODD $\times$ sex and socioemotional competence $\times$ sex. The term for the socioemotional competence $\times$ ODD $\times$ sex interaction was not significant in models predicting overall $C D$ $(\beta=0.10, p=.353)$, aggressive $\mathrm{CD}(\beta=0.12, p=$ $.442)$, and rule-breaking $\mathrm{CD}(\beta=0.07, p=.479)$ symptoms, demonstrating no sex differences for the moderation effects.

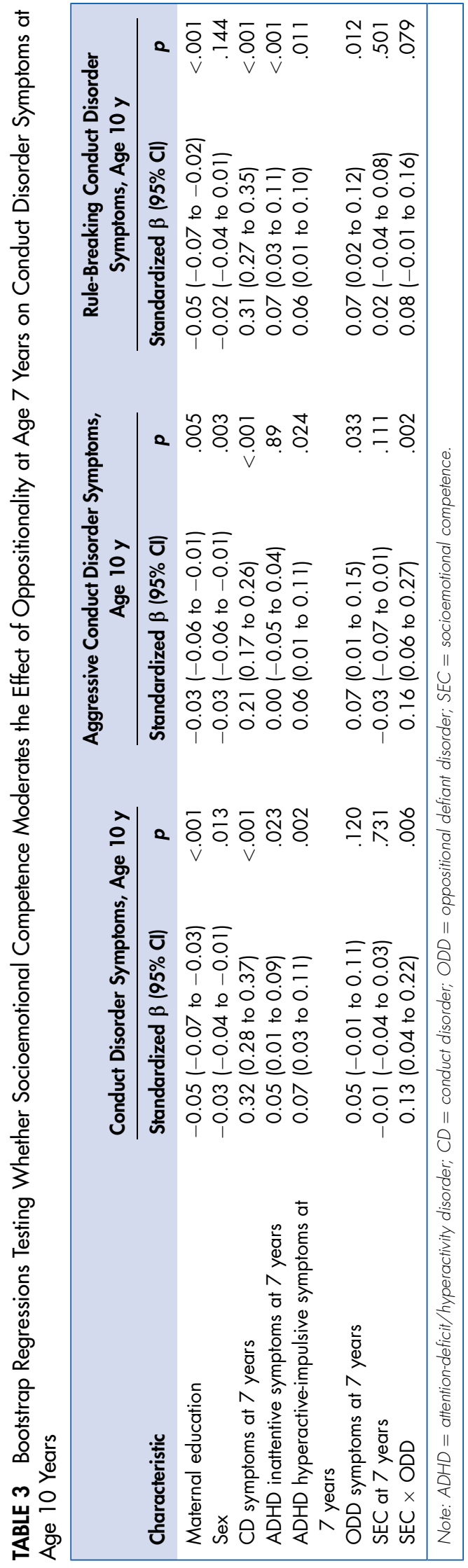




\section{DISCUSSION}

ODD symptoms are a risk factor for the subsequent development of more serious behavior problems characteristic of conduct disorder (CD). ${ }^{4}$ In a large, longitudinal UK community sample, we used parent-report data to examine whether a capacity for understanding the subjective states of others (socioemotional competence) moderated this developmental relationship. Based on clinical experience and existing literature, we hypothesized that ODD traits and socioemotional competence in middle childhood would interact in their association with CD symptoms in later childhood, such that, where socioemotional competence was poor, the association would be stronger. Regression models were used to test for this anticipated moderation, while controlling for sex, maternal education, inattention, hyperactivityimpulsivity, and CD symptoms at 7 years. In addition, we tested for potential sex differences in these associations and compared aggressive and rule-breaking $\mathrm{CD}$ symptoms. We discuss our findings, their implications for future research and clinical practice, and study limitations.

We found evidence that the developmental relationship between oppositionality at 7 years and CD symptoms at 10 years was moderated by parent-rated socioemotional competence in this large UK sample. As expected, for children with no symptoms of socioemotional impairment, there was only a weak longitudinal relationship between ODD and CD symptoms, although the relationship became increasingly strong as a function of socioemotional competence. In those with just 1 reported socioemotional competence difficulty, this relationship was stronger; and for those with multiple (2 or more) difficulties, ODD traits were a still larger risk factor for the development of subsequent CD symptoms. In our sample, this moderation effect was apparent for both boys and girls.

Our hypothesis was based on the idea that socioemotional competence helps to regulate behavior by making available an adverse experience to a child when they cause distress in others. Accordingly, we further reasoned that the moderating effect of socioemotional competence would be particularly salient for CD symptoms that most directly and obviously cause immediate suffering in others, namely overt acts of aggression such as bullying, fighting, and threatening. Our analyses offered partial support for this prediction. In separate analyses, there was a significant interaction between ODD symptoms and socioemotional competence for aggressive $\mathrm{CD}$ symptoms, whereas for rule-breaking $\mathrm{CD}$ symptoms, the moderation effect was not significant. However, formal statistical comparison of the effect sizes in these models did not show that the interaction effect for aggression was significantly bigger than for rule-breaking CD symptoms. These paradoxical findings raise the possibility that socioemotional competence does indeed play a role in moderating the relationship between ODD and subsequent rule-breaking CD symptoms, but that our study failed to detect this. Moderation effects are notoriously difficult to detect in nonexperimental studies because of considerations of statistical power, even in large samples such as ours. ${ }^{25}$ Further investigations using larger samples and more detailed measures of CD symptoms and socioemotional competence are warranted to further examine the hypothesized difference in moderation effects for aggressive and rule-breaking symptoms.

In typical development, levels of disruptive behavior, including aggression, decline during childhood, ${ }^{26}$ and our findings fit with theories that attribute this phenomenon to the child's emerging capacity for intersubjectivity. For example, Fonagy ${ }^{16}$ has argued that an increase in "mentalizing" (i.e., the ability to interpret the behavior of others as being driven by mental states) is crucial in the "unlearning" of physical aggression during typical development. Similarly, Blair's ${ }^{15}$ Integrated Emotional Systems model states that the increasing regulation of disruptive behavior in typical development is partly dependent on a child's ability to understand and to be adversely affected by the distress of other people.

Both ODD and CD are dimensional disorders, representing the extreme of traits that extend throughout the general population. ${ }^{2}$ Hence, we posit that the developmental processes that we observed in our community sample are likely to be relevant to understanding severe conduct problems described by ODD and CD diagnoses. CD can be parsed into childhood- and adolescentonset subgroups, which have partially distinct etiologies, treatment needs, and prognoses. ${ }^{1,27}$ Our study included children in middle and late childhood, so we seek to relate our findings only to the development of childhood-onset CD. Compared to its childhood-onset equivalent, adolescent-onset $\mathrm{CD}$ is less associated with dispositional risks, including impaired socioemotional competence, ${ }^{21}$ so it may be that the 
socioemotional competence moderation effect that we report is less relevant to understanding its etiology.

Our moderation model provides some insight into why a transition from ODD to childhoodonset CD is rare, ${ }^{6}$ as it suggests a protective effect of socioemotional competence. We propose that children with ODD who develop childhoodonset $C D$ may have an inferior capacity for understanding the subjective states of others compared to children with ODD who do not go onto develop CD. Supportive evidence for the hypothesis is provided by the findings that impaired socioemotional competence ${ }^{21}$ and autistic social-communication traits ${ }^{11}$ are common among children with early-onset conduct problems. Also, our findings may help to explain why fewer girls than boys progress from ODD to childhoodonset $\mathrm{CD} .{ }^{18}$ In common with other studies, ${ }^{23}$ we found that females tend to have greater socioemotional competence than males. As a result, females are more likely to benefit from the protective effects of socioemotional competence in buffering the risk posed by oppositionality.

ODD is a pivotal disorder in developmental psychopathology, which places individuals at risk for a range of concurrent and subsequent internalizing and externalizing disorders. ${ }^{5,28}$ This multifinality is of clinical concern, and future research is warranted to test whether the buffering effect of socioemotional competence that we observed for $C D$ operates with respect to other forms of subsequent psychopathology. Awareness of the moderating effects of socioemotional competence has the potential to promote a better understanding of ODD's multi-finality. That is, the current findings suggest that children with ODD and poor socioemotional competence are at risk for developing severe externalizing difficulties. It will be interesting to examine whether those children with ODD and socioemotional skills risk being on a different maladaptive trajectory not identified in the current study, for example, one characterized by internalizing difficulties. Furthermore, there is currently interest in the idea that emotional and behavioral symptoms of ODD should be distinguished. ${ }^{29}$ This is based on the discovery that, whereas ODD symptoms are highly intercorrelated and all predict disruptive behavior outcomes, only emotional symptoms (loss of temper, touchiness, anger) are uniquely predictive of emotional problems. ${ }^{28}$ On this basis the Disruptive Behavior Disorders Workgroup have proposed that, in
DSM-5, ODD symptoms be organized into "angry/irritable mood," "argumentative/defiant behavior," and "vindictiveness" clusters. ${ }^{30}$ It will be important in future analyses to test whether the moderation effect that we have found applies to each of these putative symptom dimensions. Such work may shed further light on the developmental processes that lead from ODD to more serious conduct problems, and would contribute to debates about the validity of the tripartite model of ODD.

The use of more fine-grained measures of empathy is warranted to refine our developmental moderation model, as there is behavioral and neuroanatomical evidence for a distinction between "emotional" (or "affective") and "cognitive" empathy, ${ }^{31}$ and the measure of socioemotional competence used in the current study draws on aspects of both. Clinical evidence suggests that it is impaired emotional, rather than cognitive, empathy that is relevant to aggression and antisocial behavior more generally, with a specific deficit in fear perception being especially important. ${ }^{32} \mathrm{We}$ predict that emotional empathy, particularly the capacity to empathize with others' fearful states, may underpin the protective effects of socioemotional competence that we observed, and may have an important part to play in our hypothesized differentiation between aggressive and rule-breaking behaviors. Furthermore, given our findings, it will be theoretically and clinically important to understand the causes of variability in socioemotional competence. Longitudinal studies investigating whether characteristics of the social environment (e.g., parental sensitivity, a supportive family environment) influence socioemotional competence would be of interest; as would genetically informative designs to test whether the construct measured by our socioemotional competence scale, like the one measured by the Social Communication Disorders Checklist from which it is derived, is highly heritable. ${ }^{20}$

Callous-unemotional traits are a key construct in understanding the development of a group of severely affected children with early-onset conduct problems. ${ }^{9}$ The question raised is how independent the developmental process involving socioemotional competence that we propose is from the pathway to antisocial behavior associated with callous-unemotional traits. We argue that socioemotional competence and callousunemotional traits are distinct constructs. The key characteristic of socioemotional competence 
is the capacity to notice and to correctly interpret the subjective experiences of other people, whereas callous-unemotional traits are characterized by indifference to the emotional states of others. Evidence for the validity of such a distinction is provided by the finding that callous-unemotional traits are independent of core autistic deficits in mind reading. ${ }^{33}$ Nevertheless, there is a need to investigate empirically the relationship between socioemotional competence and callous-unemotional traits; and to test whether they operate as separate moderators of the longitudinal links between ODD and CD.

Our study had several strengths, including a prospective design, the use of well-validated, clinically relevant measures, and a large sample size. Nevertheless, the current findings should be considered in the light of the following limitations. First, our data were collected by parent report, raising the issue of shared-method variance. Although this is unlikely to give rise to artifactual moderation effects, it might exaggerate the size of direct associations between variables. Our findings will be more robust if they are replicated using observational measures of social-emotional competence, so it will be important to retest our hypotheses using standardized tests of emotion recognition and empathy. Second, there was marked attrition in our community sample, with a bias favoring more socially advantaged individuals, compromising the accuracy of any prevalence estimates. However, given that the nature of our analyses is association, we argue that this attrition does not negate our findings. Indeed, recent findings from the ALSPAC cohort, which are especially pertinent to the current study as they concerned behavior problems, have shown only a marginal effect of drop-out on estimates of associations between variables. ${ }^{34}$ Furthermore, we controlled for socioeconomic

\section{REFERENCES}

1. American Psychiatric Association. Diagnostic and Statistical Manual of Mental Disorders. 4th ed, text revision. Washington, DC: American Psychiatric Association; 2000.

2. Fergusson DM, Boden JM, Horwood LJ. Classification of behavior disorders in adolescence: scaling methods, predictive validity, and gender differences. J Abnorm Psychol. 2010;119:699-712.

3. Sterba SK, Copeland W, Egger HL, Costello JE, Erkanli A, Angold A. Longitudinal dimensionality of adolescent psychopathology: testing the differentiation hypothesis. J Child Psychol Psychiatry. 2010;51:871-884.

4. Burke JD, Waldman I, Lahey BB. Predictive validity of childhood oppositional defiant disorder and conduct disorder: implications for the DSM-V. J Abnorm Psychol. 2010;119:739-751.

5. Stringaris A, Goodman R. Longitudinal outcome of youth oppositionality: irritable, headstrong, and hurtful behaviors have distinctive predictions. J Am Acad Child Adolesc Psychiatry. 2009; 48:404-412. variability, bolstering the argument that our findings are applicable across the spectrum of social advantage.

In conclusion, although ODD traits in childhood are a risk factor for the subsequent development of $\mathrm{CD}$ symptoms, the capacity to accurately perceive the perspectives and emotions of others-i.e., socioemotional competence-may buffer the risk posed by oppositionality for the development of conduct problems, including aggressive behavior. Childhood conduct problems are overdetermined, ${ }^{8}$ and recent advances in their treatment have shown the need for interventions that simultaneously address multiple risk factors. ${ }^{35}$ Our findings suggest the value of testing whether work designed to improve socioemotional competence will be a useful component of interventions aimed at reducing conduct problems in high-risk populations. $\varepsilon$

Accepted April 16, 2013.

Dr. Mandy is with University College London (UCL). Dr. Skuse is with UCL Institute of Child Health. Mr. Steer is with the Centre for Child and Adolescent Health at the University of Bristol. Dr. St Pourcain is with the School of Social and Community Medicine at the University of Bristil. Dr. Oliver is with the School of Psychology at the University of Sussex. Mr. Steer served as the statistical expert for this research.

The authors are extremely grateful to all the families who took part in this study, the midwives for their help in recruiting them, and the whole Avon Longitudinal Study of Parents and Children (ALSPAC) team, which includes interviewers, computer and laboratory technicians, clerical workers, research scientists, volunteers, managers, receptionists, and nurses. The UK Medical Research Council and the Wellcome Trust (grant 092731), and the University of Bristol provide core support for ALSPAC. This publication is the work of the authors, who will serve as guarantors for the contents of this paper.

Disclosure: Drs. Mandy, Skuse, St Pourcain, and Oliver, and Mr. Steer report no biomedical financial interests or potential conflicts of interest.

Correspondence to William Mandy, D.Clin.Psy., Research Department of Clinical, Educational and Health Psychology, University College London, UK, WC1N 6BT; e-mail: w.mandy@ucl.ac.uk 0890-8567/\$36.00/@2013 American Academy of Child and Adolescent Psychiatry

http://dx.doi.org/10.1016/i.jaac.2013.04.011
6. Rowe R, Costello EJ, Angold A, Copeland WE, Maughan B. Developmental pathways in oppositional defiant disorder and conduct disorder. J Abnorm Psychol. 2010;119:726-738.

7. Sroufe LA, Rutter M. The domain of developmental psychopathology. Child Dev. 1984;55:17-29.

8. McMahan RJ, Frick PJ, Conduct and Oppositional Disorders In: Mash JM, Barkley RA, 4th eds. Assessment of Childhood Disorders. London: Guilford Press; 2007:132-184.

9. Frick PJ, Viding E. Antisocial behavior from a developmental psychopathology perspective. Dev Psychopathol. 2009;21:1111-1131.

10. Fairchild G, Van Goozen SHM, Calder AJ, Stollery SJ Goodyer IM. Deficits in facial expression recognition in male adolescents with early-onset or adolescence-onset conduct disorder. J Child Psychol Psychiatry. 2009;50:627-636.

11. Donno R, Parker G, Gilmour J, Skuse DH. Social communication deficits in disruptive primary-school children. Br J Psychiatry. 2010;196:282-289. 
12. Olson SL, Lopez-Duran N, Lunkenheimer ES, Chang $\mathrm{H}$ Sameroff AJ. Individual differences in the development of early peer aggression: integrating contributions of self-regulation, theory of mind, and parenting. Dev. Psychopathol. 2011;23:253-266.

13. Wootton JM, Frick PJ, Shelton KK, Silverthorn P. Ineffective parenting and childhood conduct problems: the moderating role of callous-unemotional traits. J Consult Clin Psychol. 1997;65: 301-308.

14. Calkins SD, Keane SP. Developmental origins of early antisocial behavior. Dev. Psychopathol. 2009;21:1095-1109.

15. Blair RJR. The roles of orbital frontal cortex in the modulation of antisocial behavior. Brain Cogn. 2004;55:198-208.

16. Fonagy P. Towards a developmental understanding of violence. Br J Psychiatry. 2003;183:190-192.

17. Tackett JL, Krueger RF, Iacono WG, McGue M. Symptom-based subfactors of DSM-defined conduct disorder: evidence for etiologic distinctions. J Abnorm Psychol. 2005;114:483-487.

18. Rowe R, Maughan B, Pickles A, Costello EJ, Angold A. The relationship between DSM-IV oppositional defiant disorder and conduct disorder: findings from the Great Smoky Mountains Study. J Child Psychol Psychiatry. 2002;43:365-373.

19. Goodman R, Ford T, Richards H, Gatward R, Meltzer H. The Development and Well-Being Assessment: description and initial validation of an integrated assessment of child and adolescent psychopathology. J Child Psychol Psychiatry. 2000;41:645-655.

20. Skuse DH, Mandy WPL, Scourfield J. Measuring autistic traits: heritability, reliability and validity of the Social and Communication Disorders Checklist. Br J Psychiatry. 2005;187:568-572.

21. Oliver BR, Barker ED, Mandy WPL, Skuse DH, Maughan B. Social cognition and conduct problems: a developmental approach. J Am Acad Child Adolesc Psychiatry. 2011;50:385-394.

22. Bishop DV. Development of the Children's Communication Checklist (CCC): a method for assessing qualitative aspects of communicative impairment in children. J Child Psychol Psychiatry. 1998;39:879-891.

23. Skuse DH, Mandy W, Steer C, Miller LL, Goodman R, Lawrence $\mathrm{K}$, et al. Social communication competence and functional adaptation in a general population of children: preliminary evidence for sex-by-verbal IQ differential risk. J Am Acad Child Adolesc Psychiatry. 2009;48:128-137.
24. Galobardes B, McCormack VA, McCarron P, Howe LD, Lynch J, Lawlor DA, et al. Social inequalities in height: persisting differences today depend upon height of the parents. PLoS One. 2012;7: e29118.

25. Aguinis H, Beaty JC, Boik RJ, Pierce CA. Effect size and power in assessing moderating effects of categorical variables using multiple regression: a 30-year review. J Appl Psychol. 2005;90: 94-107.

26. Tremblay RE. Developmental origins of disruptive behavior problems: the "original $\sin ^{\prime}$ hypothesis, epigenetics and their consequences for prevention. J Child Psychol Psychiatry. 2010;51: 341-367.

27. Moffitt TE, Caspi A. Childhood predictors differentiate life-course persistent and adolescence-limited antisocial pathways among males and females. Dev Psychopathol. 2001;13:355-375.

28. Stringaris A, Goodman R. Three dimensions of oppositionality in youth. J Child Psychol Psychiatry. 2009;50:216-223.

29. Drabick DAG, Gadow KD. Deconstructing oppositional defiant disorder: clinic-based evidence for an anger/irritability phenotype. J Am Acad Child Adolesc Psychiatry. 2012;51:384-393.

30. American Psychiatric Association. Proposed Revision. Available at: http://www.dsm5.org/ProposedRevisions/Pages/ proposedrevision.aspx?rid=106\# Accessed November 12, 2012.

31. Blair RJR. Fine cuts of empathy and the amygdala: dissociable deficits in psychopathy and autism. Q J Exp Psychol (Hove). 2008; 61:157-170.

32. Marsh AA, Blair RJR. Deficits in facial affect recognition among antisocial populations: a meta-analysis. Neurosci Biobehav Rev. 2008;32:454-465.

33. Rogers J, Viding E, Blair RJ, Frith U, Happé F. Autism spectrum disorder and psychopathy: shared cognitive underpinnings or double hit? Psychol Med. 2006;36:1789-1798.

34. Wolke D, Waylen A, Samara M, Steer C, Goodman R, Ford T, et al. Selective drop-out in longitudinal studies and non-biased prediction of behavior disorders. Br J Psychiatry. 2009;195: 249-256.

35. Butler S, Baruch G, Hickey N, Fonagy P. A randomized controlled trial of multisystemic therapy and a statutory therapeutic intervention for young offenders. J Am Acad Child Adolesc Psychiatry. 2011;50:1220-1235. 